\title{
结合空间注意力机制与光流特征的微表情识别方法
}

\author{
刘德志1)，梁正友 ${ }^{1,2)^{*}}$ ，孙宇 ${ }^{1)}$ \\ ${ }^{1)}$ (广西大学计算机与电子信息学院 南宁 530004) \\ 2) (广西多媒体通信与网络技术重点实验室 南宁 530004) \\ (zhyliang@gxu.edu.cn)
}

\begin{abstract}
摘 要: 针对微表情运动的局部性问题, 提出一种将深度学习的空间注意力机制与微表情光流特征相结合的微表情 识别自动方法. 首先, 采用帧差法识别缺少峰值帧标记的微表情样本的峰值帧; 然后, 利用 TV- $L_{1}$ 光流法提取微表情 起始帧与峰值帧之间的光流水平、垂直分量图，并根据光流的水平、垂直分量图导出对应的光流应变模式图; 将 3 个光流图以通道叠加的方式连接起来, 构成微表情的光流特征图; 最后, 在 Inception 模块搭建的卷积神经网络中设 计了一种包含可学习参数的空间注意力单元, 使模型在特征提取过程中能够更加关注存在微表情运动的区域. 在空 间注意力单元中利用 $3 \times 3$ 和 $7 \times 7$ 这 2 种大小的卷积核进行空间注意力的推断，使模型能够综合地考虑不同尺度卷积 核的注意力推断结果. 实验结果表明, 该方法在 MEGC2019 综合微表情数据集上的识别准确率达到 0.788 , 优于已有 的微表情识别方法.
\end{abstract}

关键词：微表情识别；深度学习；空间注意力机制；光流法；卷积神经网络；情绪分类

中图法分类号: TP391.41ＤOI: 10.3724/SP.J.1089.2021.18569

\section{Micro-Expression Recognition Method Based on Spatial Attention Mechanism and Optical Flow Features}

\author{
Liu Dezhi ${ }^{1)}$, Liang Zhengyou ${ }^{1,2)^{*}}$, and Sun $\mathrm{Yu}^{1)}$ \\ 1) (School of Computer and Electronics Information, Guangxi University, Nanning 530004) \\ ${ }^{2)}$ (Guangxi Key Laboratory of Multimedia Communications and Network Technology, Nanning 530004)
}

\begin{abstract}
In order to solve the locality problem of micro-expression movement, an automatic micro-expression recognition method based on spatial attention mechanism of deep learning and optical flow features of micro-expressions is proposed. First, the frame difference method is used to identify apex frame for some micro-expression samples lack of apex frame labels. Then, TV- $L_{1}$ optical flow method is used to extract the horizontal and vertical components map of optical flow between the onset frame and the apex frame of micro-expression, and the corresponding optical flow strain pattern map is derived according to the horizontal and vertical components of optical flow. The three optical flow maps are connected in the way of channel superposition to form an optical flow characteristic map of micro-expression. Finally, a kind of spatial attention unit with learnable parameters is designed in the convolutional neural network built by the Inception module, which makes the model pay more attention to the regions with micro-expression motion in the feature extraction process. In the spatial attention unit, two convolution kernels of $3 \times 3$ and $7 \times 7$ are used for spatial attention inference, so that the model can comprehensively consider the attention inference results of different scale convolution kernels. Experiments on the MEGC2019 comprehensive micro-expression
\end{abstract}

收稿日期: 2020-08-10; 修回日期: 2021-06-16. 基金项目: 国家自然科学基金(61763002). 刘德志(1993一), 男, 硕士研究生, 主 要研究方向为计算机视觉、深度学习; 梁正友(1968一), 男, 博士, 教授, CCF 会员, 论文通讯作者, 主要研究方向为计算机视觉、无 线传感器网络、并行分布式计算、人工智能; 孙宇(1981一), 女, 博士, 讲师, 主要研究方向为智能算法、图像识别、数据挖掘. 
datasets show that the accuracy of the method is 0.788 , which is better than the existing automatic micro-expression recognition method.

Key words: micro-expression recognition; deep learning; spatial attention mechanism; optical flow method; convolutional neural network; emotion classification

微表情是一种由内心真实情绪激发所产生, 可以准确地反映人内心真情实感的面部表情, 能 够作为测谎的重要依据, 在心理治疗、商业谈判和 国家安全等领域有着广阔的应用前景.

相关研究表明, 微表情具有以下 3 个特点 ${ }^{[1]}$. (1) 运动强度较弱. 仅凭肉眼很难察觉到微表情的 发生. (2) 存在时间极短. 通常情况下持续时间仅 为 $1 / 25 \sim 1 / 3 \mathrm{~s}$. (3) 运动具有局部性. 微表情只是在 一个极短的时间内由部分面部区域的一些很小的 肌肉收缩运动所产生, 因此在多数情况下仅能在 面部的局部区域观察到微表情的运动. 这 3 个特点 给微表情的自动识别工作造成了一定的困难.

近年来, 一些微表情自动识别方法试图从微 表情运动强度较弱和微表情运动的局部性这 2 个 特点人手以提高识别准确率. 针对运动强度较弱 的问题, 常用的方法是利用运动放大算法 ${ }^{[2]}$ 放大微 表情的运动 ${ }^{[3]}$. 这种方法存在的问题是必须对运动 放大倍数等参数进行严格的控制, 以避免运动放 大后产生的伪影对识别效果的影响. $\mathrm{Li}$ 等 ${ }^{[4]}$ 的工作 表明, 放大倍数为 $[8,16]$ 时, 对识别准确率的提升 帮助较大. 放大倍数过小, 识别效果的提升并不明 显; 而放大倍数太大则会产生过多的伪影, 导致识 别准确率降低. 对面部进行感兴趣区域(region of interest, ROI)划分则是目前常用于解决微表情运动 局部性问题的一种方法. Polikovsky 等 ${ }^{[5]}$ 根据面部 动作编码系统 (facial action coding system, FACS $)^{[6]}$ 定义的动作单元(action unit, $A U$ ), 将面部划分成 12 个 ROI, 并根据 12 个 ROI 的三维方向梯度直方 图特征对微表情进行分类. Liu 等 ${ }^{[7]}$ 则通过 66 个面 部特征点将人脸分成了 36 个 ROI, 每个 ROI 包含 若干个 $\mathrm{AU}$, 根据各个 $\mathrm{ROI}$ 的主方向光流特征来识 别微表情.

以手工的方式对面部进行 ROI 划分可以忽略 与微表情运动无关的区域, 在一定程度上减少了 呪余的特征. 但是不同情感类别的微表情涉及的 AU 不同, 即使是精心地设计了面部 ROI 的划分, 也难以保证每个 ROI 都存在有助于微表情分类的 特征, 特征冗余的问题依然存在. 且对面部进行
ROI 划分之后, 由于各个区域的形状不规则和大 小不统一等原因, 难以使用目前流行的深度学习 技术, 只能采用较为烦琐的手工特征提取方式进 行识别.

针对微表情运动的局部性问题, 本文提出一 种结合空间注意力机制和峰值帧光流特征的微表 情自动识别方法. 首先, 通过帧差法确定无峰值帧 标记的微表情样本的峰值帧; 然后, 将 TV- $L_{1}$ 光流 法 ${ }^{[8]}$ 提取的峰值帧光流的水平、垂直分量图和导出 的光流应变模式图以通道叠加的方式连接起来, 组成微表情的光流特征图; 最后, 在 Inception 模 块 ${ }^{[9]}$ 构建的卷积神经网络 (convolutional neural networks, CNN) 模型中设计一种包含可学习参数 的空间注意力单元(spatial attention unit, SAU), 帮 助模型在提取光流特征图上的微表情特征时, 学 会关注面部存在微表情运动的 ROI. 在 SAU 中, 利用 $3 \times 3$ 和 $7 \times 7$ 这 2 种大小的卷积核进行空间注 意力的推断, 使模型能够综合地考虑不同尺度卷 积核的注意力推断结果. 实验表明, 本文方法的准 确率优于已有的微表情自动识别方法.

\section{1 相关工作}

\section{1 基于深度学习的微表情自动识别方法}

微表情是一个面部肌肉运动的过程, 因此当 前一些基于深度学习的识别方法试图通过对微表 情视频帧序列进行时空特征编码实现微表情的自 动识别. 目前常用于对微表情进行时空特征编码 的深度学习技术主要有 $\mathrm{CNN}+$ 长短期记忆网络 (long short-term memory, LSTM)混合模型 ${ }^{[10-11]}$, 3D $\mathrm{CNN}^{[12-13]}$ 等. 由于微表情运动较微弱, 高速摄像 机采集到的微表情视频帧序列存在大量的圥余帧. 此外, 对帧序列进行时空特征编码存在计算量过 大、硬件性能要求较高的问题.

相关研究表明 ${ }^{[14-15]}$, 帧序列中记录了面部运 动最为强烈的峰值帧, 可以为微表情的分类任务 提供足够的信息. 因此, 近年来出现了大量的利用 深度学习技术提取峰值帧特征进行微表情识别的 
方法. Gan 等 ${ }^{[16]}$ 以帧序列的起始帧为参考帧，提取 峰值帧的光流特征训练所提出的 OFF-ApexNet, 得到了 0.746 的微表情识别准确率. Liong 等 ${ }^{[17]}$ 从 峰值帧的光流特征当中导出光流应变模式特征, 并与光流特征相结合, 使识别准确率得到了提高. Zhou 等 ${ }^{[18]}$ 提出的双流 Inception 网络, 2 个流的输人 分别是峰值帧光流的水平、垂直分量图, 在全连接 层以特征融合的方式将 2 个流提取到的特征连接 起来, 利用 Softmax 层进行分类. Wang 等 ${ }^{[19]}$ 在 ResNet 的残差单元中设计了特殊的微注意单元, 使网络在特征提取的过程中更加关注发生微表情 运动的区域. Xia 等 ${ }^{[20]}$ 受到类激活图特征可视化技 术的启发, 提出了一个不包含学习参数的注意力 单元, 以避免领域偏移对模型学习效果的影响. 上 述工作表明利用微表情峰值帧提供的信息就可以 获得较好的识别准确率, 与当前的一些对微表情 视频帧序列进行时空特征编码的识别方法相比, 大大减少了计算量和运行时间.

\section{2 空间注意力机制}

空间注意力机制是一种借鉴了人类视觉系统 注意力机制的模型特征优化方法, 其原理是通过 加权的方式增强图像上包含有效分类特征的区域, 并对包含无用特征的区域进行抑制, 防止模型学 习到错误的分类信息. 近年来的一些研究表明, 空 间注意力机制能够有效地提升模型在各种深度学 习任务中的性能. Jaderberg 等 ${ }^{[21]}$ 提出的空间变换 网络能够通过学习的方式对输人图像进行各种形 式的空间变换, 从而突出图像上包含重要特征的 区域. Woo 等 ${ }^{[22]}$ 首先利用通道方向的平均池化和 最大池化聚合中间特征映射各个通道的信息，然 后通过卷积核尺寸为 $7 \times 7$ 的卷积操作推断图像上 各个区域的重要程度. Rodriguez 等 ${ }^{[23]}$ 则融合了不 同深度卷积层的多尺度信息获取表示各个区域权 重的空间注意力图.

由于微表情运动具有局部性特点, 大多数面 部区域并不存在有效的分类特征, 只有少数存在 微表情运动的 ROI 才能提供有助于微表情分类的 信息. 而其他的区域, 如眼部区域, 存在每个微表 情类别都有可能出现的眨眼动作, 甚至会提供错 误的分类信息. 针对这个问题, 本文在模型中设计 了一种具有学习能力的 SAU, 对 Inception 模块输 出的特征映射进行优化, 使模型在特征提取的过 程中更加关注存在微表情运动的 ROI, 从而提高 模型的识别性能.

\section{3 光流法及光流应变模式}

光流法是一种常用于对视频中的物体进行运 动估计的方法. 光流法通过光流场反映视频中的 物体在连续 2 帧中的运动. 光流场是一个二维的运 动场, 表示图像上每个像素运动的大小和方向. 光 流场比原始灰度图像更清楚地反映了面部发生微 表情运动的区域, 使模型学习到更高层次的微表 情运动和形变特征的同时, 更有利于 SAU 推断存 在微表情运动的 ROI.

光流法基于 3 个假设性原则：(1) 亮度恒定原 则, 同一物体相邻 2 帧的亮度不变; (2) 时间连续 原则, 同一物体相邻 2 帧的位置变化微小; (3) 空 间一致性原则, 相邻像素点具有相似的运动.

设 $I(x, y, t)$ 为 $t$ 时刻图像位置 $(x, y)$ 处的亮度 值. 该像素点在时间 $\mathrm{d} t$ 内移动距离为 $(\mathrm{d} x, \mathrm{~d} y)$, 由 亮度恒定原则可以认为该像素的值不变, 即

$$
I(x, y, t)=I(x+\mathrm{d} x, y+\mathrm{d} y, t+\mathrm{d} t)
$$

将式(1)右边取泰勒级数近似, 去掉一般项并除以 $\mathrm{d} t$ 得到光流方程 $\frac{\partial I}{\partial x} \frac{\mathrm{d} x}{\mathrm{~d} t}+\frac{\partial I}{\partial y} \frac{\mathrm{d} y}{\mathrm{~d} t}+\frac{\partial I}{\partial t}=0$. 光流法的 目的就是计算出相邻 2 帧之间每个像素点的瞬时 速度矢量 $\boldsymbol{p}=\left[p=\frac{\mathrm{d} x}{\mathrm{~d} t}, q=\frac{\mathrm{d} y}{\mathrm{~d} t}\right]^{\mathrm{T}}$, 从而估计出连续 2 帧中物体的运动趋势.

应变模式用于衡量物体在外力作用下的形变 程度. 相关研究表明 ${ }^{[24]}$, 应变模式仅与面部的形 变有关, 不易受光照条件、面部遮盖物或头部的微 小运动等因素的影响, 在微表情的检测和识别任 务中有较好的表现 ${ }^{[25]}$.

设 $\boldsymbol{u}=[u, v]^{\mathrm{T}}$ 表示三维空间中面部表情形变导 致的位移在二维图像上的投影, 则可用柯西张量 表示面部肌肉组织的形变大小, 即

$$
\boldsymbol{\varepsilon}=\frac{1}{2}\left[\nabla \boldsymbol{u}+(\nabla \boldsymbol{u})^{\mathrm{T}}\right]
$$

其中, $\nabla$ 表示 $\boldsymbol{u}$ 对进行求导. 可以将式(2)的二维 应变张量展开成矩阵形式

$$
\boldsymbol{\varepsilon}=\left[\begin{array}{ll}
\varepsilon_{x x}=\frac{\partial u}{\partial x} & \varepsilon_{x y}=\frac{1}{2}\left(\frac{\partial u}{\partial x}+\frac{\partial v}{\partial y}\right) \\
\varepsilon_{y x}=\frac{1}{2}\left(\frac{\partial v}{\partial x}+\frac{\partial u}{\partial y}\right) & \varepsilon_{y y}=\frac{\partial v}{\partial y}
\end{array}\right] .
$$

其中, $\varepsilon_{x x}$ 和 $\varepsilon_{y y}$ 为正应变分量; $\varepsilon_{x y}$ 和 $\varepsilon_{y x}$ 为切应变 分量. 由于每个应变分量都是位移矢量 $\boldsymbol{u}=[u, v]^{\mathrm{T}}$ 的 函数, 因此可以将光流表示的速度矢量 $\boldsymbol{p}=[p, q]^{\mathrm{T}}$ 
近似为位移矢量 $\boldsymbol{u}=[u, v]^{\mathrm{T}}$. 其中,

$$
\begin{aligned}
& p=\frac{\mathrm{d} x}{\mathrm{~d} t} \approx \frac{\Delta x}{\Delta t}=\frac{u}{\Delta t} ; u=p \Delta t, \\
& q=\frac{\mathrm{d} y}{\mathrm{~d} t} \approx \frac{\Delta y}{\Delta t}=\frac{v}{\Delta t} ; v=q \Delta t .
\end{aligned}
$$

将 2 帧之间的时间间隔 $\Delta t$ 看做一个常数后， 就可以利用速度矢量来近似计算位移矢量的偏导 数, 即

$$
\begin{aligned}
& \frac{\partial u}{\partial x}=\frac{\partial p}{\partial x} \Delta t ; \frac{\partial u}{\partial y}=\frac{\partial p}{\partial y} \Delta t \\
& \frac{\partial v}{\partial x}=\frac{\partial q}{\partial x} \Delta t ; \frac{\partial v}{\partial y}=\frac{\partial q}{\partial y} \Delta t
\end{aligned}
$$

并通过中心差分法分别计算出式(3)(4)表示的位移矢 量的偏导数, 即

$$
\frac{\partial u}{\partial x}=\frac{u(x+\Delta x)-u(x-\Delta x)}{2 \Delta x} \approx \frac{p(x+\Delta x)-p(x-\Delta x)}{2 \Delta x},
$$

$$
\frac{\partial v}{\partial y}=\frac{v(y+\Delta y)-v(y-\Delta y)}{2 \Delta y} \approx \frac{q(y+\Delta y)-q(y-\Delta y)}{2 \Delta y} .
$$

$\Delta x$ 和 $\Delta y$ 通常为 $1 \sim 3$ 个像素, 本文的取值为 2 个像 素， $\frac{\partial u}{\partial y}$ 和 $\frac{\partial v}{\partial x}$ 可以通过类似的方法进行计算. 由于 微表情运动过程中肌肉运动可能包含多个方向, 因此采用 $\varepsilon_{\mathrm{m}}=\sqrt{\varepsilon_{x x}^{2}+\varepsilon_{y y}^{2}+\varepsilon_{x y}^{2}+\varepsilon_{y x}^{2}}$ 计算每个像素 的应变大小. 利用光流矢量导出的应变模式也称 为光流应变模式.

\section{2 本文方法}

本文方法分为获取微表情运动峰值帧位置、提 取光流特征和分类识别 3 个步骤，如图 1 所示.

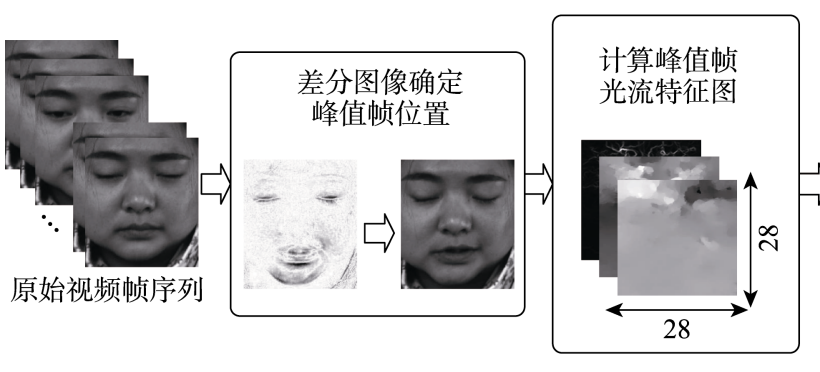

空间注意力InceptionNet提取微表情特征并分类

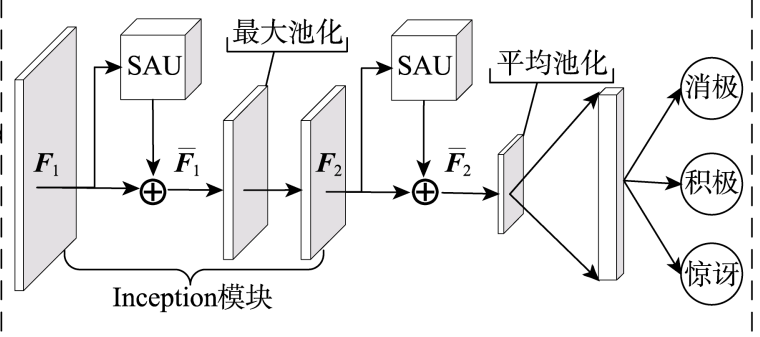

图 1 本文方法流程

\section{1 获取微表情运动峰值帧位置}

在提取微表情峰值帧的光流特征前，首先需 要解决部分微表情样本缺少峰值帧标记的问题. 本文借鉴了通过帧差法 ${ }^{[26]}$ 进行运动目标检测和跟 踪的原理, 通过差分图像确定缺少峰值帧标记的 微表情样本的峰值帧位置. 设 $V=\left\{v_{1}, v_{2}, \cdots, v_{k}\right\}$ 为 待检测的微表情视频帧序列集合， $k$ 为待检测的 样本数; 设 $v_{n}=\{f(x, y, 1), f(x, y, 2), \cdots, f(x, y, t)\}$ 为 第 $n$ 个待检测的帧序列, $f(i, j, z)$ 表示第 $z$ 帧位置 $(i, j)$ 处的像素灰度值; 则第 $z$ 帧图像的差分图 $d$ 位置 $(i, j)$ 处的像素值的计算公式为

$$
d(i, j, z)=f(i, j, z)-r(i, j) .
$$

其中, $r(i, j)$ 为面部无表情的参考帧位置 $(i, j)$ 处的 像素灰度值. 由于峰值帧是微表情运动过程中面 部变化最大的一帧, 因此差分图像在峰值帧附近 能够检测到更明显的面部变化，如图 2 所示.

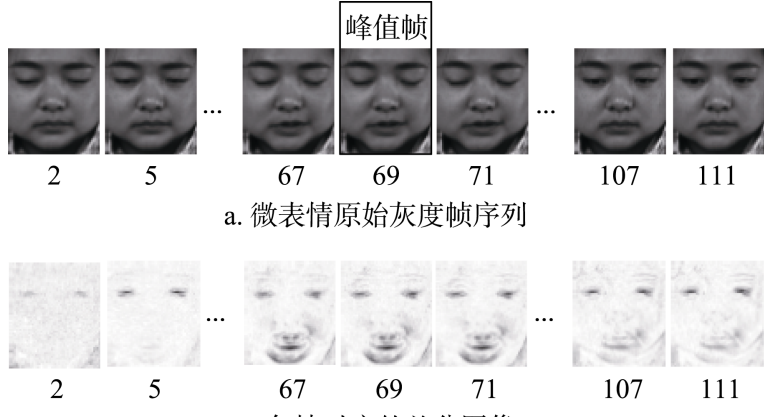

b. 各帧对应的差分图像

图 2 微表情原始灰度帧序列及对应的差分图

计算出帧序列中每帧的差分图像后，根据

$$
A_{z}=\sqrt[2]{\sum_{j=0}^{y} \sum_{i=0}^{x} d(i, j, z)^{2}}
$$

评估每帧的面部形变程度, 将 $A_{z}$ 值最大的一帧确 定为该样本的峰值帧.

由于每个样本的起始帧和结束帧都是中性的 
无表情帧, 因此在峰值帧的测定过程中分别以起 始帧和结束帧为参考帧，将 2 种参考帧测定结果的 均值定为该样本的峰值帧位置, 以减小误差.

\section{2 提取光流特征}

Liong 等 ${ }^{[27]}$ 对 5 种光流法进行了比较, 结果表 明 TV- $L_{1}$ 光流法在微表情识别任务当中有更好的 表现，因此本文采用 TV- $L_{1}$ 光流法对微表情进行运 动估计. 即以微表情视频帧序列的起始帧为参考 帧，利用 OpenCV 提供的光流计算函数 DualTVL1OpticalFlow_create 对起始帧与峰值帧进 行光流估计. 计算结果是一个二维矢量场, 即与峰 值帧图像的空间大小相等的双通道矩阵，分别表 示每个像素运动的水平和垂直分量.

本文还利用中心差分法计算光流的水平和垂 直分量对应的光流应变模式图. 因此, 每个微表情 样本可以得到如图 3 所示光流的水平、垂直分量图 和光流应变模式图. 最后将每个样本的 3 个光流图 以通道叠加的方式连接起来, 组成微表情的光流 特征图, 将大小调整至 28×28 并进行标准化处理 后, 输人模型进行训练. 为了获取更多的训练样 本，增强模型的泛化能力并减少过拟合现象，本文 还计算了每个样本峰值帧前后 2 帧的光流图并加 人训练集当中.
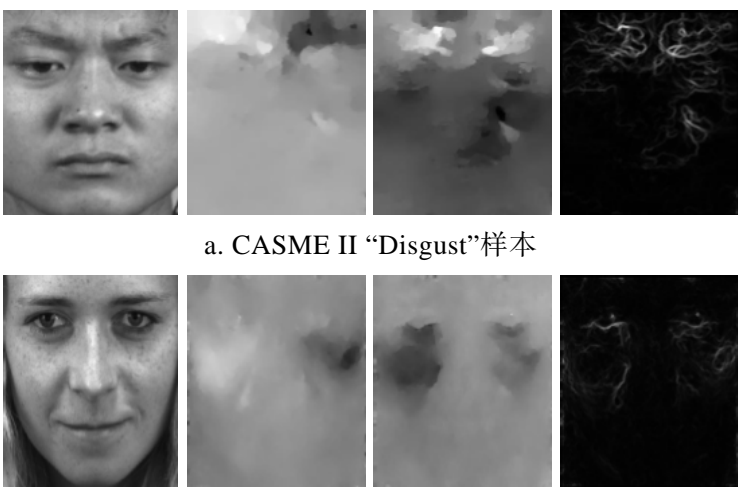

a. CASME II “Disgust”样本

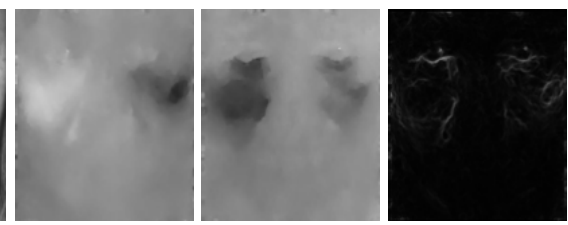

b. SMIC-HS “Surprise”样本

图 3 微表情峰值帧原始灰度图和 3 个光流特征图

\section{3 网络结构设计}

\subsubsection{SAU}

本节将具体介绍 SAU 的实现方式, 其结构如 图 4 所示. 对第 $l$ 个卷积层输出的大小为 $C \times H \times W$ 中间特征映射矩阵 $\boldsymbol{F}_{l}, \mathrm{SAU}$ 的目标是推导出大小 为 $H \times W$ 的二维空间注意力映射矩阵.

为了生成二维的空间注意力映射矩阵, 需要 聚合 $\boldsymbol{F}_{l}$ 各个通道的空间特征，将通道维数降为 1 . Woo 等 ${ }^{[22]}$ 证明了同时采用最大池化和平均池化 2

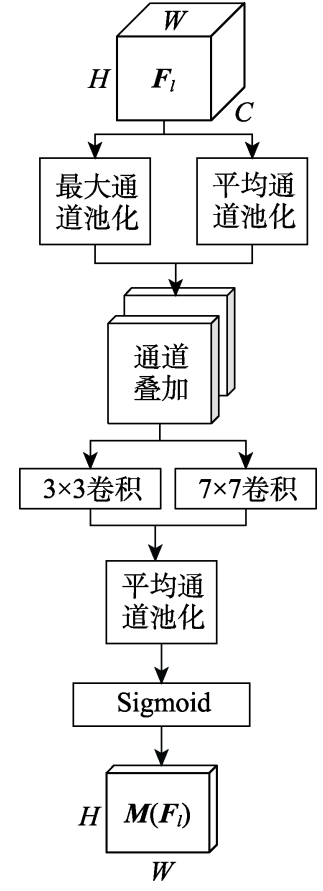

图 4 SAU 结构示意图

种方式聚合通道信息更有利于模型进行注意力推 断. 因此在 SAU 中同时利用了这 2 种池化操作对 $\boldsymbol{F}_{l}$ 进行通道降维, 分别生成 $\boldsymbol{F}_{l}$ 的平均通道池化、最 大通道池化特征描述符.

之后将 2 个特征描述符以通道叠加的方式连 接起来, 通过卷积操作推断 $\boldsymbol{F}_{l}$ 各个区域的重要性. 对 $\boldsymbol{F}_{l}$ 进行卷积操作的卷积核是参数可学习的矩阵. 在模型的学习过程当中, 通过调节 SAU 中的卷积 核参数来调整空间注意力映射各位置的权重值, 即对要强调或抑制的图像区域进行编码. SAU 的 结构借鉴了 Inception 模块利用不同大小的卷积核 来增强模型对不同尺度特征适应性的思想，利用 $3 \times 3$ 和 $7 \times 7$ 这 2 种大小的卷积核推导 2 种空间注 意力映射 $\boldsymbol{M}\left(\boldsymbol{F}_{l}\right)_{3 \times 3}$ 和 $\boldsymbol{M}\left(\boldsymbol{F}_{l}\right)_{7 \times 7}$, 使 SAU 能够利用 多个尺度的信息，综合考虑不同区域特征的重要 程度, 如

$$
\boldsymbol{M}\left(\boldsymbol{F}_{l}\right)_{n \times n}=f^{n \times n}\left[P_{\text {ave }}\left(\boldsymbol{F}_{l}\right) ; P_{\max }\left(\boldsymbol{F}_{l}\right)\right]
$$

其中, $f^{n \times n}$ 表示卷积核大小为 $n \times n$ 卷积操作; $P_{\text {ave }}\left(\boldsymbol{F}_{l}\right)$ 和 $P_{\text {max }}\left(\boldsymbol{F}_{l}\right)$ 分别表示通道维度的平均池化 和最大池化. 最后, 通过平均通道池化来融合 2 种 注意力 $\boldsymbol{M}\left(\boldsymbol{F}_{l}\right)_{3 \times 3}$ 和 $\boldsymbol{M}\left(\boldsymbol{F}_{l}\right)_{7 \times 7}$ 推断结果, 并通过 Sigmoid 激活函数将结果规范化到 $[0,1]$, 即

$$
\boldsymbol{M}\left(\boldsymbol{F}_{l}\right)=\sigma\left[P_{\text {ave }}\left(\boldsymbol{M}\left(\boldsymbol{F}_{l}\right)_{3 \times 3} ; \boldsymbol{M}\left(\boldsymbol{F}_{l}\right)_{7 \times 7}\right)\right]
$$

其中, $\sigma$ 表示 Sigmoid 激活函数. 式(6)中的 $\boldsymbol{M}\left(\boldsymbol{F}_{l}\right)$ 即为推导出的空间注意力映射, 表示 $\boldsymbol{F}_{l}$ 各个区域 
元素的权重, 即模型应该关注 $\boldsymbol{F}_{l}$ 的哪些区域. 将 $\boldsymbol{M}\left(\boldsymbol{F}_{l}\right)$ 与 $\boldsymbol{F}_{l}$ 的每个通道进行相同空间位置的元素 相乘，增强 $\boldsymbol{F}_{l}$ 各个通道相同区域的特征之后，将 结果与 $\boldsymbol{F}_{l}$ 按照矩阵加法规则相加, 生成优化后的 特征映射矩阵为 $\bar{F}_{l}=\left[M\left(F_{l}\right) \otimes F_{l}\right] \oplus F_{l}$. 其中, $\otimes$ 表示 $\boldsymbol{M}\left(\boldsymbol{F}_{l}\right)$ 与 $\boldsymbol{F}_{l}$ 各通道的同位置元素相乘; $\oplus$ 表 示矩阵的同位置元素相加.

\subsection{2 空间注意力 InceptionNet}

CNN 通过对输人图像进行逐像素的卷积操作, 生成相应的特征映射来表示输人图像. 设 CNN 的 第 $l$ 个卷积层输出的特征映射位置 $(i, j)$ 处的值为 $y_{i, j}^{l}=f\left(c_{i, j}^{l}+b^{l}\right)$. 其中, $b^{l}$ 为偏置值; $f(x)$ 表示 激活函数; $c_{i, j}^{l}$ 为第 $l$ 层输人图像 $x^{l}$ 位置 $(i, j)$ 处的 卷积计算结果, 即 $c_{i, j}^{l}=\sum_{a=0}^{n-1} \sum_{b=0}^{n-1} w_{a, b}^{l} x_{(i+a),(j+b)}^{l}$. 其中, $n$ 为卷积核的尺寸; $x_{i, j}^{l}$ 为第 $l$ 层输人图像位置 $(i, j)$ 处的值; $w_{a, b}^{l}$ 为第 $l$ 层卷积核位置 $(a, b)$ 处的 值. 卷积核的尺寸 $n$ 是对模型性能有重要影响的 参数，常见的卷积核尺寸有 $3 \times 3,5 \times 5$ 和 $7 \times 7$ 等. 大的卷积核有利于提取大范围的全局信息，而小 的卷积核在提取小范围的局部区域特征时更有优 势. 本文利用 Inception 模块来构建所提出的空间 注意力 InceptionNet 网络(spatial attention InceptionNet, SAIN), 使模型在训练过程中自适应地选 择合适的卷积核尺寸进行特征提取, 增强网络对 不同尺度特征的适应能力.

Inception 模块由 $1 \times 1,3 \times 3$ 和 $5 \times 5$ 这 3 个卷积 核尺寸不同的卷积层和最大池化层共 4 个分支组 成, 分别对上层传人的特征映射进行卷积和池化 操作, 如图 5 所示. 但直接将 4 个分支生成的特征 映射以通道叠加的方式连接起来会存在特征映射 的通道维度过多的问题, 导致整个网络的计算量

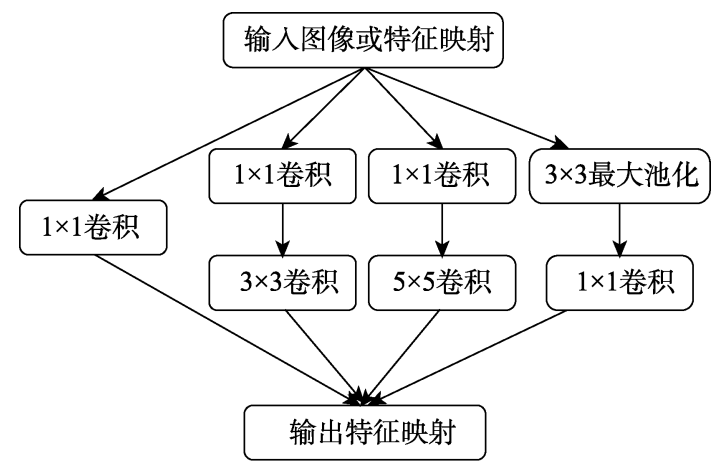

图 5 Inception 模块结构示意图
过大. 因此, Inception 模块在 $3 \times 3,5 \times 5$ 的卷积层 和最大池化层前增加了 $1 \times 1$ 的卷积层.

对微表情的光流特征图进行特征提取的 SAIN 由 2 个 Inception 模块堆叠而成, 如图 1 虚线框中所 示. 每个 Inception 模块输出的特征映射 $\boldsymbol{F}_{l}$ 都利用 第 2.3.1 节提出的 SAU 进行优化, 生成优化后的特 征映射 $\overline{\boldsymbol{F}}_{l}$. 在将 $\overline{\boldsymbol{F}}_{l}$ 传人下一层之前, 还需要对其 进行池化操作，以减少特征维度. 第 1 个 Inception 模块优化后的特征映射采用最大池化, 从而能够 在突出重要特征的同时消除冗余信息. 第 2 个 Inception 模块优化后的特征映射采用平均池化, 平均池化通过求池化窗口内像素均值的方式对特 征映射进行空间降维, 使每个像素都会对降维结 果产生影响, 保留了更多的图像特征. 最后将平均 池化结果输人长度为 256 的全连接层, 并利用 Softmax 层进行分类. 模型具体参数如表 1 所示.

表 1 SAIN 具体参数设置

\begin{tabular}{lcc}
\hline \multicolumn{1}{c}{ 层 } & 卷积核数量 & 输出大小 \\
\hline Inception Module 1 & 12 & $28 \times 28 \times 48$ \\
SAU 1 & & $28 \times 28 \times 48$ \\
Max Pooling & & $14 \times 14 \times 48$ \\
Inception Module 2 & 24 & $14 \times 14 \times 96$ \\
SAU 2 & & $14 \times 14 \times 96$ \\
Average Pooling & & $7 \times 7 \times 96$ \\
Flatten Layer & & $256 \times 1$ \\
Softmax Layer & & $3 \times 1$ \\
\hline
\end{tabular}

\section{3 实验及结果分析}

\section{1 实验数据集、评估方式和实验配置}

3.1.1 实验数据集

本文在 MEGC2019 综合微表情数据集上对提 出的方法进行验证. MEGC2019 综合微表情数据集 从 CASME II ${ }^{[28]}, \mathrm{SAMM}^{[29]}$ 和 SMIC-HS ${ }^{[30]} 3$ 个自发 式微表情数据集中选取了 442 个样本. 其中来自 CASME II, SAMM 和 SMIC-HS 的样本数量分别为 145，133 和 164, 并根据样本的情绪标签将它们分 成了 3 个类别: 积极 (positive)、消极 (negative) 和惊 讶(surprise). 具体的划分方式如表 2 所示. 在 3 个 数据集中, CASME II 和 SAMM 这 2 个数据集都提 供了样本的起始帧、峰值帧、结束帧位置和由 FACS 编码的 AU 标记; 而 SMIC-HS 未提供样本的峰值 帧和 AU 标记, 其峰值帧采用第 2.1 节描述的方法 测定. 
表 2 MEGC2019 详细信息

\begin{tabular}{ccll}
\hline 类别 & 数量 & \multicolumn{1}{c}{ 情绪标签 } & \multicolumn{1}{c}{ 原数据集 } \\
\hline & & 厌恶(disgust) & CASME II, SAMM \\
& & 压抑(repression) & CASME II \\
& & 䟠视(contempt) & SAMM \\
\multirow{2}{*}{ negative } & \multirow{2}{*}{250} & 愤怒(anger) & SAMM \\
& & 悲伤(sadness) & SAMM \\
& & 恐惧(fear) & SAMM \\
& & 消极(negative) & SMIC-HS \\
\hline \multirow{2}{*}{ positive } & \multirow{2}{*}{109} & 快乐(happiness) & CASME II, SAMM \\
& & 积极(positive) & SMIC-HS \\
\hline \multirow{2}{*}{ surprise } & \multirow{2}{*}{83} & 惊讶(surprise) & CASME II, SAMM, \\
\hline & & & SMIC-HS \\
\hline
\end{tabular}

\subsection{2 评估方式}

为了验证本文方法的有效性, 采用复合数据 库评估(composite database evaluation, $\mathrm{CDE}$ )和保持 数据库评估(holdout-database evaluation, HDE)对所 提出的方法进行验证.

在 CDE 验证中, 将来自 3 个数据集的样本放 在一起执行留一受试交叉验证(leave one subject out, LOSO). 每轮的 LOSO 验证将其中一名受试者 的样本作为测试集, 其余受试者的样本作为训练 集. LOSO 验证准确率的计算公式为 $a=\frac{1}{k} \sum_{i=1}^{k} a_{i}$. 其中, $k$ 为受试者数量, MEGC2019 综合微表情数 据集包含 68 名受试者的微表情样本, 因此需要执 行 68 轮验证, 即 $k=68 ; a_{i}$ 为第 $i$ 轮验证准确率.

HDE 验证是一种跨数据集验证方式. 在 HDE 验证中, 每次将一个数据集的微表情样本作为测 试集, 剩下 2 个数据集的样本作为训练集. 最后, 根据在 3 个测试数据集上的识别准确率等指标对 方法进行评估. HDE 验证除了证明本文方法的有 效性之外, 还能探究不同数据集的微表情样本之 间是否具有相同或相似的特征, 即模型在其他 2 个 数据集上学习到的微表情特征是否能用于其他的 数据集.

由于 MEGC2019 综合微表情数据集存在各个 类别的样本数量不均衡的问题, 在 CDE 验证和 HDE 验证中, 对本文方法的 2 个指标非加权 $F_{1}$ 得 分(unweighted $F_{1}$-score, $\mathrm{UF}_{1}$ ) 和非加权平均召回率 (unweighted average recall, UAR)进行比较, 以更 好地对所提方法的性能进行评估. UF 1 和 UAR 的计 算公式分别为

$$
\begin{gathered}
\mathrm{UF}_{1}=2 \times \frac{\sum_{i=1}^{M} \frac{P_{i} \times R_{i}}{P_{i}+R_{i}}}{M} ; \\
\mathrm{UAR}=\frac{1}{M} \sum_{i=1}^{M} \frac{\sum_{j=1}^{k} \mathrm{TP}_{i}^{j}}{M \times \sum_{j=1}^{k} \mathrm{TP}_{i}^{j}+\sum_{j=1}^{k} \mathrm{FN}_{i}^{j}} .
\end{gathered}
$$

其中, $M$ 表示类别数量, 本文将微表情分为积极、 消极和惊讶 3 个类别, 因此 $M=3 ; P$ 和 $R$ 分别表 示根据分类结果的真正例(true positive, TP)、假正例 (false positive, FP)和假负例(false negative, FN)计算 出的精确率和召回率. $P$ 与 $R$ 的计算公式分别为

$$
\begin{gathered}
P=\sum_{i=1}^{M} \frac{\sum_{j=1}^{k} \mathrm{TP}_{i}^{j}}{M \times \sum_{j=1}^{k} \mathrm{TP}_{i}^{j}+\sum_{j=1}^{k} \mathrm{FP}_{i}^{j}}, \\
R=\sum_{i=1}^{M} \frac{\sum_{j=1}^{k} \mathrm{TP}_{i}^{j}}{M \times \sum_{j=1}^{k} \mathrm{TP}_{i}^{j}+\sum_{j=1}^{k} \mathrm{FN}_{i}^{j}} .
\end{gathered}
$$

\subsection{3 实验配置}

实验的操作系统环境为 Centos6.5, 利用 TensorFlow1.15.0 和 Keras2.3.1 完成模型的搭建, 编程语言为 Python3.6, 模型训练的主要硬件设备 为 NVIDIA TESLA T4. 模型的学习速率为 0.000 , epoch $=100$, dropout 层的丢弃率为 0.3 , 模型训练优 化器为 Adam, 损失函数采用交叉熵.

\section{2 峰值帧测定结果}

为检验帧差法的峰值帧检测效果，在带有真 实峰值帧标记的 CASME II 和 SAMM 数据集上进 行了实验, 并将帧差法的峰值帧检测平均有效误

\begin{tabular}{|c|c|c|c|}
\hline 方法 & 测试数据集 & 平均帧长/帧 & MAE/帧 \\
\hline Liong 等 ${ }^{[31]}$ & CASME II & 66.9 & 13.6 \\
\hline $\mathrm{Li}$ 等 ${ }^{[15]}$ & CASME II & 66.9 & 11.8 \\
\hline \multirow{2}{*}{ 本文 } & CASME II & 66.9 & 11.8 \\
\hline & SAMM & 74.3 & 12.3 \\
\hline
\end{tabular}
差(mean absolute error, MAE)与近年来的一些峰值 帧测定方法进行了比较, 如表 3 所示.

表 3 峰值帧测定方法对比

表 3 中的 MAE 定义为测量峰值帧和真实峰值 帧之间的平均帧误差，即 


$$
\text { MAE }=\frac{1}{N} \sum_{i=1}^{n}\left|e_{i}\right| .
$$

其中, $e_{i}$ 为第 $i$ 个样本的测量峰值帧和真实峰值帧 之间的误差; $N$ 为测试数据集中的微表情样本数. 实验结果显示, 本文采用的帧差法的峰值帧检测 MAE 约为 12 帧, 在较为合理的范围之内.

\section{3 实验结果分析}

\subsubsection{CDE 验证}

在 MEGC2019 综合微表情数据集上, 将本文 方法与已有的微表情识别方法进行 68 轮 LOSO 准 确率对比, 如表 4 所示. 复现的方法相关实验参数 均按照原文中公布的最佳实验参数进行设置. 本 文方法每轮 LOSO 验证所需的时间约为 $100 \mathrm{~s}, 68$ 轮 LOSO 需要大约 $6800 \mathrm{~s}$.

表 4 本文方法与前沿微表情自动识别方法比较

\begin{tabular}{lccc}
\hline \multicolumn{1}{c}{ 方法 } & 准确率 & $\mathrm{UF}_{1}$ & $\mathrm{UAR}$ \\
\hline RNMA $^{[19]}$ & 0.613 & 0.508 & 0.493 \\
ATNet $^{[11]}$ & 0.693 & 0.631 & 0.613 \\
OFF-ApexNet $^{[16]}$ & 0.746 & 0.719 & \\
Dual-InceptionNet $^{[18] *}$ & 0.762 & 0.695 & 0.677 \\
RCN-A $^{[20] *}$ & 0.766 & 0.708 & 0.711 \\
STSTNet $^{[17]}$ & $\mathbf{0 . 7 6 9}$ & $\mathbf{0 . 7 3 5}$ & $\mathbf{0 . 7 6 1}$ \\
本文 & $\mathbf{0 . 7 8 8}$ & $\mathbf{0 . 7 2 1}$ & $\mathbf{0 . 7 1 7}$ \\
\hline
\end{tabular}

注. 粗体表示最优和次优数据, 带 $*$ 号的为复现的实验结果.

由表 4 可以看出, 本文方法在 LOSO 验证中的 准确率达到了 0.788, 优于已有的识别方法. Wang 等 ${ }^{[19]}$ 的 RNMA 方法在 ResNet10 模型中添加了特殊 的微注意单元, 使模型在提取微表情峰值帧原始 灰度图特征的过程中更加关注面部发生变化的区 域. 但是由于微表情的运动较微弱, 峰值帧与面部 无表情的差异很小, 在原始灰度图上较难判断哪 个面部区域发生了微表情运动, 因此识别准确率 相对较低. 而 ATNet 方法 ${ }^{[11]}$ 在利用 ResNet10 提取 微表情峰值帧原始灰度图特征的基础上, 利用 LSTM 对峰值帧前后 32 帧的光流特征进行时域特 征编码，为模型的决策提供辅助信息.

上述 2 种方法都是利用 CNN 提取峰值帧原始 灰度图特征. 而近年来提出的一些结合深度学习 技术与光流特征的识别方法普遍都实现了更高的 识别准确率. 其中, Gan 等 ${ }^{[16]}$ 和 Zhou 等 ${ }^{[18]}$ 设计的 模型都采用了类似的双流结构, 分别将峰值帧光 流的水平、垂直分量图作为 2 个流的输人, 在全连 接层将 2 个流提取的特征连接起来后, 利用 Softmax 层进行分类. Liong 等 ${ }^{[17]}$ 将峰值帧光流的
水平、垂直分量图和光流应变模式图以通道叠加的 方式连接起来, 组成微表情的光流特征图对所提 出的 STSTNet 模型进行训练. 同时, STSTNet 采用 了 3 个流的网络结构, 每个流的卷积层都包含了数 量不等的卷积核, 以此避免样本较少造成的过拟 合问题，因此在 $U_{1}$ 和 UAR2 项指标上更有优势. Xia 等 ${ }^{[20]}$ 设计的注意力单元中并不存在任何的可训练 参数, 因此模型无法在模型训练的反向传播过程 中对各区域的权重进行调整.

而本文方法在 Inception 模块搭建的 CNN 中设 计了包含学习参数的 SAU, 使模型在提取峰值帧 光流特征图上的微表情特征时, 更加关注由微表 情导致的面部发生变化的区域. 在 SAU 中利用 $3 \times 3$ 和 $7 \times 7$ 这 2 种大小的卷积核进行空间注意力 的推断, 使模型能够综合地考虑不同尺度卷积核 的注意力推断结果. 同时, 在 SAU 中的卷积层中 包含了一些可学习的参数, 使 SAU 在训练过程中 不断地对各个区域特征的权重进行调整和优化, 能够更准确地进行注意力的推断. 此外, 峰值帧光 流的水平、垂直分量图和光流应变模式图反映了面 部发生微表情运动和形变的区域, 比原始灰度图 更能突出面部的细微变化, 使模型能更准确地推 断在微表情的持续过程中面部发生运动的区域, 实现了更高的识别准确率.

图 6 给出了 SAIN 在 MEGC2019 综合微表情 数据集以及 3 个子数据集的分类结果混淆矩阵. 可 以看出, SAIN 能够较准确地识别消极类别, 而对 积极和惊讶类别的识别准确率相对较低. 这是由 于 MEGC2019 综合微表情数据集存在样本类别分 布不均衡的问题, 在 442 个微表情样本中包含了大 量的消极类别. 特别是 SAMM数据集, 在 133 个样 本当中仅有 26 个积极类别和 15 个惊讶类别, 造成 该数据集不同类别识别准确率差异较大的现象最 为明显.

\subsubsection{HDE 验证}

表 5 所示为本文方法的 HDE 验证结果与前沿 的微表情识别方法的比较. 其中, RNMA 与 ATNet 方法的 HDE 验证结果来自文献[11], 文中仅记录 了这 2 种方法的 $U_{1}$ 和 UAR. 而 Dual-InceptionNet, STSTNet 和 RCN-A 这 3 种方法的原文并未进行 $\mathrm{HDE}$ 验证, 因此本文参考这些方法的作者在 GitHub 上共享的实验代码进行了 HDE 验证. 相 关实验参数按照原文中公布的最佳实验参数进行 设置.

此外, 还可以观察到 SAMM 和 SMIC-HS 作为 


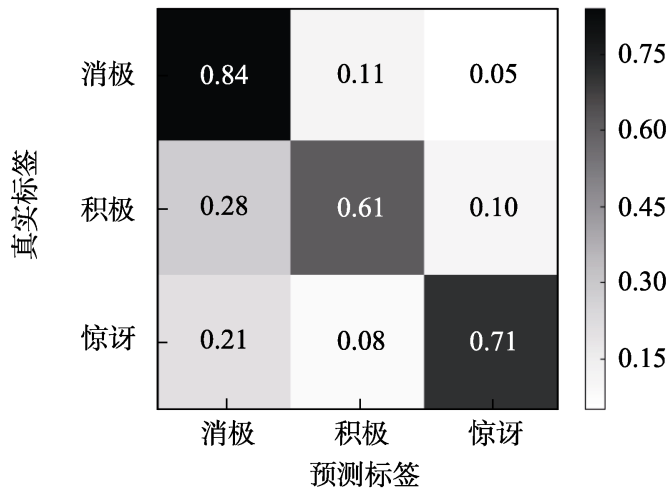

a. MEGC2019
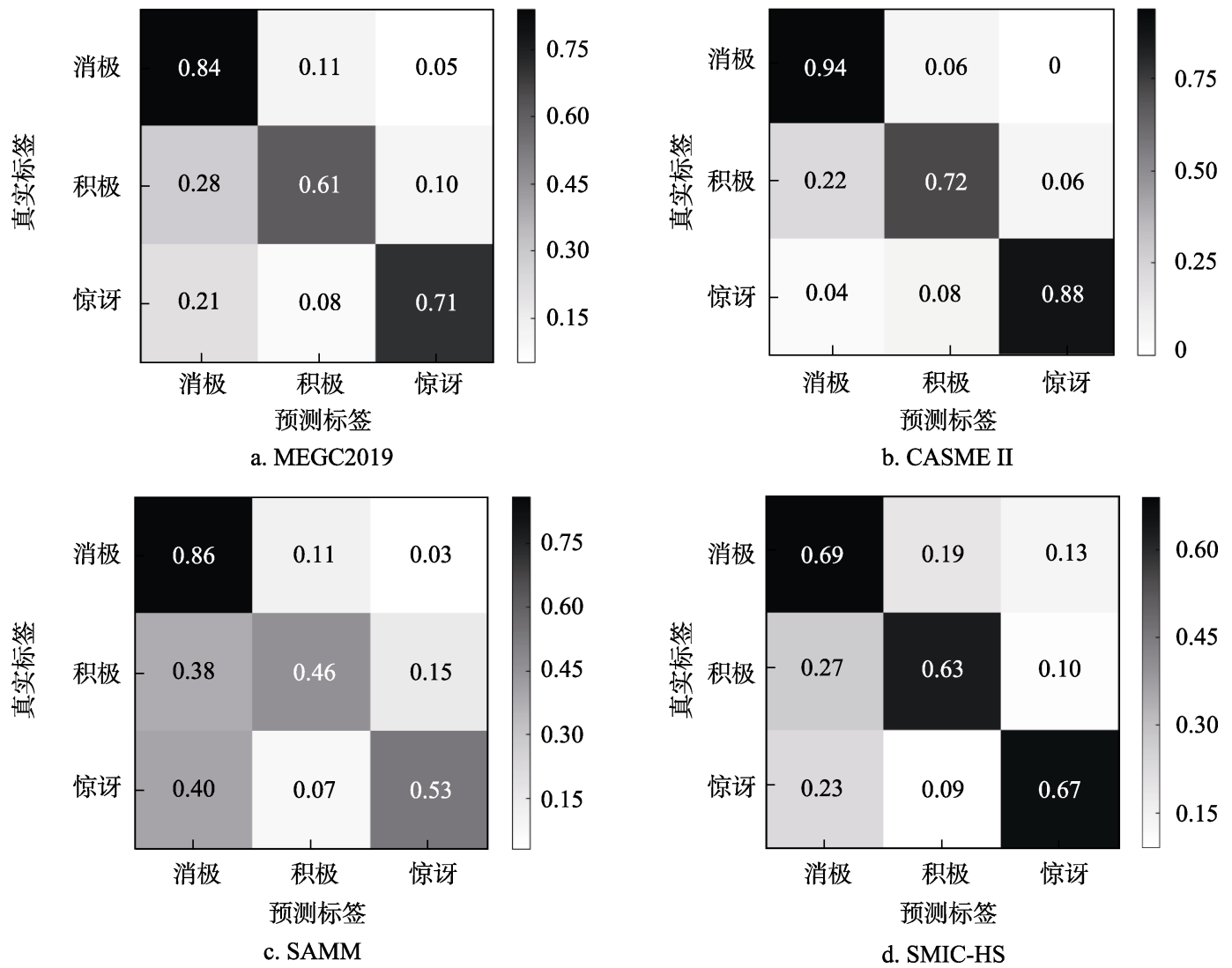

图 6 SAIN 在 MEGC2019 识别结果混淆矩阵

表 5 CASME II 作为测试集的 HDE 验证结果

\begin{tabular}{|c|c|c|c|c|c|c|c|c|c|}
\hline \multirow{2}{*}{ 方法 } & \multicolumn{3}{|c|}{ CASME II } & \multicolumn{3}{|c|}{ SAMM } & \multicolumn{3}{|c|}{ SAMIC-HS } \\
\hline & LOSO 准确率 & $\mathrm{UF}_{1}$ & UAR & LOSO 准确率 & $\mathrm{UF}_{1}$ & UAR & LOSO 准确率 & $\mathrm{UF}_{1}$ & UAR \\
\hline RNMA $^{[19]}$ & & 0.338 & 0.369 & & 0.383 & 0.380 & & 0.354 & 0.372 \\
\hline $\operatorname{ATNet}^{[11]}$ & & 0.631 & 0.643 & & 0.450 & 0.458 & & 0.503 & 0.524 \\
\hline Dual-InceptionNet ${ }^{[18]} *$ & 0.821 & 0.729 & 0.723 & 0.632 & 0.576 & 0.628 & 0.622 & 0.607 & 0.599 \\
\hline STSTNet ${ }^{[17] *}$ & 0.848 & 0.823 & 0.818 & 0.624 & 0.588 & 0.616 & 0.554 & 0.540 & 0.533 \\
\hline $\mathrm{RCN}-\mathrm{A}^{[20] *}$ & 0.841 & 0.824 & 0.820 & 0.602 & 0.651 & 0.555 & 0.561 & 0.535 & 0.555 \\
\hline 本文(无 SAU) & 0.807 & 0.766 & 0.753 & 0.669 & 0.581 & 0.619 & 0.579 & 0.557 & 0.542 \\
\hline 本文 & 0.876 & 0.849 & 0.839 & 0.684 & 0.609 & 0.653 & 0.628 & 0.617 & 0.601 \\
\hline
\end{tabular}

注. 粗体表示最优和次优数据, 带*号的为复现的实验结果.

测试集时的各项指标相对较低. 造成这个现象的 原因主要有 2 个: (1) 属于消极类别的 contempt, anger, sadness 和 fear 微表情样本仅存在于 SAMM 数据集中, 因此将 SAMM 作为测试集时, 模型较 难从训练集中学习到这几类微表情的特征; (2) SMIC-HS 数据集的人脸区域相对较小，且样本清晰 度相对较差, 对光流运动估计造成了一定的影响.

\section{4 光流特征和 SAU 对识别效果的影响}

本文在相同的实验环境下，对未加人 SAU 的 原始模型 UNSAIN_OF、利用原始灰度图进行训练 的空间注意力模型 SAIN_GRAY 和利用光流特征
图进行训练的注意力模型 SAIN_OF 进行了对比. 表 6 对 3 组模型的 LOSO 准确率、 $\mathrm{UF}_{1}$ 和 UAR 进 行了比较，同时计算了 3 个子数据集的 $\mathrm{UF}_{1}$ 和 UAR. 从表 6 数据可以看出, 利用光流特征图进行 训练的 UNSAIN_OF 和 SAIN_OF 在完整数据集和 3 个子数据集上的各个指标均优于 SAIN_GRAY. 其主要原因在于由光流的水平、垂直分量和光流应 变模式图组成的光流特征图上强调了面部发生变 化的区域, 因此更有利于模型对微表情进行特征 提取. 而SAIN_OF 模型中的 SAU 能够使模型更加 关注这些发生了微表情运动和形变的区域, 因此 
表 6 微表情光流特征和 SAU 对识别效果的影响

\begin{tabular}{|c|c|c|c|c|c|c|c|c|c|}
\hline \multirow{2}{*}{ 模型 } & \multicolumn{3}{|c|}{ MEGC2019 } & \multicolumn{2}{|c|}{ CASME II } & \multicolumn{2}{|c|}{ SAMM } & \multicolumn{2}{|c|}{ SMIC-HS } \\
\hline & 准确率 & $\mathrm{UF}_{1}$ & UAR & $\mathrm{UF}_{1}$ & UAR & $\mathrm{UF}_{1}$ & UAR & $\mathrm{UF}_{1}$ & UAR \\
\hline UNSAIN_OF & 0.752 & 0.688 & 0.671 & 0.827 & 0.818 & 0.567 & 0.548 & 0.630 & 0.620 \\
\hline SAIN_GRAY & 0.562 & 0.436 & 0.434 & 0.545 & 0.527 & 0.381 & 0.368 & 0.371 & 0.391 \\
\hline SAIN_OF & 0.788 & 0.721 & 0.717 & 0.856 & 0.847 & 0.623 & 0.618 & 0.652 & 0.651 \\
\hline
\end{tabular}

准确率和其他指标与无注意力机制的 UNSAIN_OF 模型相比有了一定程度的提高.

本文进一步利用了梯度加权类激活图特征可 视化技术 ${ }^{[32]}$ (gradient-weighted class activation mapping, Grad-CAM), 以探究微表情的光流特征和提 出的 SAU 在特征提取的过程中对模型的影响. 首 先, 提取 UNSAIN_OF, SAIN_GRAY 和 SAIN_OF 这 3 组模型最后一个 Inception 模块输出的特征映 射计算 Grad-CAM 图; 然后, 将其上采样后与对应 的峰值帧灰度图进行叠加, 并与反映面部形变情 况的光流应变模式图进行比较, 如图 7 所示. 图 7 中 $p$ 值为模型的 Softmax 层输出的判别矢量中, 将 该样本判定为预测类别的概率. 可以看出, UNSAIN_OF 和 SAIN_GRAY 这 2 组模型在提取图 像特征时, 可能会将大部分注意力放在颈部、眼部 或面部无明显运动等与微表情识别无关的区域. 而在 SAIN_OF 模型的 Grad-CAM 图中, 模型能够 将更多的注意力放在光流应变模式图上反映面部 形变的区域. 从这几组 Grad-CAM 图中可以看出, 所提出的 SAIN_OF 模型能从光流特征图中提取出 更有利于分类的特征, 且提出的 SAU 能够帮助模 型在特征提取的过程中, 更加关注存在微表情运 动和形变特征的区域, 从而提高识别准确率.

\section{4 结 语}

针对微表情运动的局部性问题, 本文提出了 一种结合空间注意力机制与光流特征的微表情自 动识别方法. 本文方法通过在 Inception 模块构成 的 CNN 中设计了具有学习能力的 SAU 实现空间注 意力机制, 使模型在提取由峰值帧光流的水平、垂 直分量图和光流应变模式图构成的光流特征图上 的微表情特征时, 能够学会关注图像上包含重要 微表情运动特征的区域. 在 SAU 中, 利用 $3 \times 3$ 和 $7 \times 7$ 大小的卷积核进行空间注意力的推断, 使模 型能够综合地考虑不同尺度卷积核的注意力推断 结果. 结果表明, 本文方法的准确率达到了 0.788 , 优于已有的识别方法.
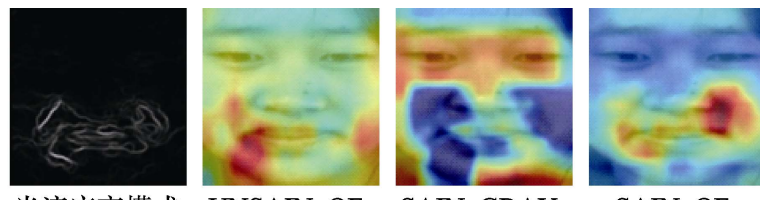

光流应变模式 UNSAIN_OF SAIN_GRAY SAIN OF 情绪标签: 压抑 预测类别: 消极 预测类别: 消极 预测类别: 消极 类别: 消极

$p: 0.540$ $p: 0.870$ $p: 0.608$

a. CASME II 数据集, 样本编号 sub02, EP02_04f

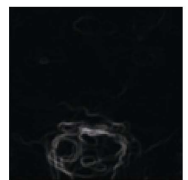

光流应变模式 情绪

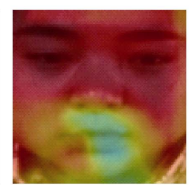
UNSAIN_OF SAI

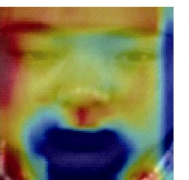

SAIN_GRAY 绪标签: 惊讶 预测类别: 消极 预测类别: 积极 预测类别: 惊讶 类别: 惊讶 $p: 0.833$

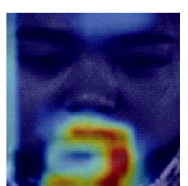

SAIN_OF b. CASME II 数据集, 样本编号 sub05, EP12_03f
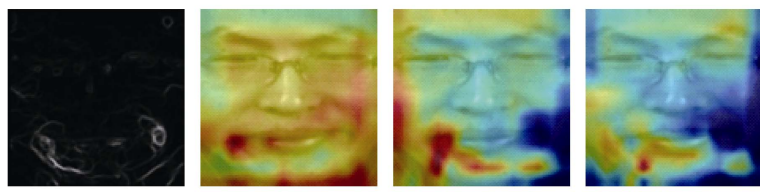

光流应变模式 UNSAIN_OF SAIN_GRAY

SAIN_OF 情绪标签: 快乐 预测类别: 消极 预测类别: 惊讶 预测类别: 积极 类别: 积极 $\quad p: 0.705$ $p: 0.866$

$p: 0.565$

c. CASME II 数据集, 样本编号 sub14, EP09_04

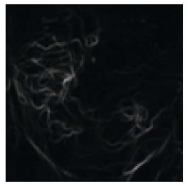

光流应变模式 标签: 戻恶 类别: 消极

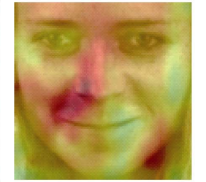

UNSAIN_OF

UNSAIN_OF SAIN_GRAY
预测类别: 消极 预测类别: 消极 $p: 0.884$

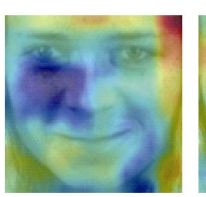
p: 0.922

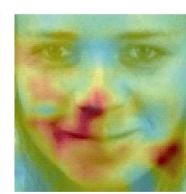

SAIN_OF $p: 0.954$

d. SAMM 数据集, 样本编号 011,011_02_1

图 7 部分微表情样本的 Grad-CAM 图

但是, 本文研究工作仍然存在一些问题. 例 如, 数据类别分布不平衡造成了模型无法有效学 习到积极与惊讶这 2 个类别的特征, 使这 2 个类别 的识别率偏低. 因此, 下一步的研究中, 可以考虑 通过类别重采样的方式减少类别分布严重不均衡 对识别准确率的影响. 此外, 利用迁移学习的技 术, 在样本数量较多的宏表情数据集上对模型进 行预训练, 以解决当前微表情样本较为稀缺导致 的模型过拟合问题也是今后的研究方向. 


\section{参考文献(References):}

[1] Ben Xianye, Yang Mingqiang, Zhang Peng, et al. Survey on automatic micro expression recognition methods[J]. Journal of Computer-Aided Design \& Computer Graphics, 2014, 26(9): 1385-1395(in Chinese)

(变晛烨, 杨明强, 张鹏, 等. 微表情自动识别综述 $[\mathrm{J}]$. 计算 机辅助设计与图形学学报, 2014, 26(9): 1385-1395)

[2] Wu H Y, Rubinstein M, Shih E, et al. Eulerian video magnification for revealing subtle changes in the world[J]. ACM Transactions on Graphics, 2012, 31(4): Article No.65

[3] Liu Ruhan, Xu Dan. Video amplification and deep learning in micro-expression recognition[J]. Journal of Computer-Aided Design \& Computer Graphics, 2019, 31(9): 1535-1541(in Chinese)

(刘汝涵, 徐丹. 视频放大和深度学习在微表情识别任务上 的应用[J]. 计算机辅助设计与图形学学报, 2019, 31(9): 1535-1541)

[4] Li X B, Hong X P, Moilanen A, et al. Towards reading hidden emotions: a comparative study of spontaneous micro-expression spotting and recognition methods[J]. IEEE Transactions on Affective Computing, 2018, 9(4): 563-577

[5] Polikovsky S, Kameda Y, Ohta Y, et al. Facial micro-expression detection in hi-speed video based on facial action coding system[J]. IEICE Transactions on Information and Systems, 2013, 96(1): 81-92

[6] Ekman P, Friesen W V. Facial action coding system (FACS): a technique for the measurement of facial action[M]. Palo Alto: Consulting Psychologists Press, 1978

[7] Liu Y J, Zhang J K, Yan W J, et al. A main directional mean optical flow feature for spontaneous micro-expression recognition[J]. IEEE Transactions on Affective Computing, 2016, 7(4): 299-310

[8] Zach C, Pock T, Bischof H. A duality based approach for realtime TV- $L_{1}$ optical flow[C] //Proceedings of Joint Pattern Recognition Symposium. Heidelberg: Springer, 2007: 214-223

[9] Szegedy C, Liu W, Jia Y Q, et al. Going deeper with convolutions[C] //Proceedings of the IEEE Conference on Computer Vision and Pattern Recognition. Los Alamitos: IEEE Computer Society Press, 2015: 1-9

[10] Khor H Q, See J, Phan R C W, et al. Enriched long-term recurrent convolutional network for facial micro-expression recognition[C] //Proceedings of the 13th IEEE International Conference on Automatic Face \& Gesture Recognition. Los Alamitos: IEEE Computer Society Press, 2018: 667-674

[11] Peng M, Wang C Y, Bi T, et al. A novel apex-time network for cross-dataset micro-expression recognition[C] //Proceedings of the 8th International Conference on Affective Computing and Intelligent Interaction. Los Alamitos: IEEE Computer Society Press, 2019: 1-6

[12] Peng M, Wang C Y, Chen T, et al. Dual temporal scale convolutional neural network for micro-expression recognition[J].
Frontiers in Psychology, 2017, 8: 1745

[13] Li J, Wang Y D, See J, et al. Micro-expression recognition based on 3D flow convolutional neural network[J]. Pattern Analysis and Applications, 2019, 22(4): 1331-1339

[14] Liong S T, See J, Wong K S, et al. Less is more: micro-expression recognition from video using apex frame[J]. Signal Processing: Image Communication, 2018, 62: 82-92

[15] Li Y T, Huang X H, Zhao G Y. Can micro-expression be recognized based on single apex frame?[C] //Proceedings of the 25th IEEE International Conference on Image Processing. Los Alamitos: IEEE Computer Society Press, 2018: 3094-3098

[16] Gan Y S, Liong S, Yau W, et al. OFF-ApexNet on micro-expression recognition system[J]. Signal Processing-Image Communication, 2019, 74: 129-139

[17] Liong S T, Gan Y S, See J, et al. Shallow triple stream three-dimensional CNN (STSTNet) for micro-expression recognition[C] //Proceedings of the 14th IEEE International Conference on Automatic Face \& Gesture Recognition. Los Alamitos: IEEE Computer Society Press, 2019: 1-5

[18] Zhou L, Mao Q R, Xue L Y, et al. Dual-inception network for cross-database micro-expression recognition[C] //Proceedings of the 14th IEEE International Conference on Automatic Face \& Gesture Recognition. Los Alamitos: IEEE Computer Society Press, 2019: 1-5

[19] Wang C Y, Peng M, Bi T, et al. Micro-attention for micro-expression recognition[J]. Neurocomputing, 2020, 410: 354-362

[20] Xia Z Q, Peng W, Khor H Q, et al. Revealing the invisible with model and data shrinking for composite-database micro-expression recognition[J]. IEEE Transactions on Image Processing, 2020, 29: 8590-8605

[21] Jaderberg M, Simonyan K, Zisserman A, et al. Spatial transformer networks[C] //Proceedings of the 28th International Conference on Neural Information Processing Systems. Cambridge: MIT Press, 2015: 2017-2025

[22] Woo S, Park J, Lee J Y, et al. CBAM: convolutional block attention module[C] //Proceedings of the European Conference on Computer Vision. Heidelberg: Springer, 2018: 3-19

[23] Rodriguez P, Velazquez D, Cucurull G, et al. Pay attention to the activations: a modular attention mechanism for fine-grained image recognition[J]. IEEE Transactions on Multimedia, 2020, 22(2): 502-514

[24] Manohar V, Goldgof D B, Sarkar S, et al. Facial strain pattern as a soft forensic evidence[C] //Proceedings of the IEEE Workshop on Applications of Computer Vision. Los Alamitos: IEEE Computer Society Press, 2007: 42

[25] Liong S T, See J, Phan R C W, et al. Spontaneous subtle expression detection and recognition based on facial strain[J]. Signal Processing: Image Communication, 2016, 47: 170-182

[26] Ju J G, Xing J S. Moving object detection based on smoothing three frame difference method fused with RPCA[J]. Multimedia Tools and Applications, 2019, 78(21): 29937-29951

[27] Liong S T, Gan Y S, Zheng D N, et al. Evaluation of the spa- 
tio-temporal features and GAN for micro-expression recognition system[J]. Journal of Signal Processing Systems, 2020, 92(1): 705-725

[28] Yan W J, Li X B, Wang S J, et al. CASME II: an improved spontaneous micro-expression database and the baseline evaluation[J]. PLoS One, 2014, 9(1): e86041

[29] Davison A K, Lansley C, Costen N, et al. SAMM: a spontaneous micro-facial movement dataset[J]. IEEE Transactions on Affective Computing, 2018, 9(1): 116-129

[30] Li X B, Pfister T, Huang X H, et al. A spontaneous micro-expression database: inducement, collection and base-
line[C] //Proceedings of the 10th IEEE International Conference and Workshops on Automatic Face and Gesture Recognition. Los Alamitos: IEEE Computer Society Press, 2013: 1-6

[31] Liong S T, See J, Wong K S, et al. Automatic apex frame spotting in micro-expression database[C] //Proceedings of the 3rd IAPR Asian Conference on Pattern Recognition. Los Alamitos: IEEE Computer Society Press, 2015: 665-669

[32] Selvaraju R R, Cogswell M, Das A, et al. Grad-CAM: visual explanatiowns from deep networks via gradient-based localization[J]. International Journal of Computer Vision, 2020, 128(2): 336-359 